\title{
Influence of hemispheric white matter lesions and migraine characteristics on cortical thickness and volume
}

\author{
Hedvig Komáromy ${ }^{1}$, Mingchen He², Gábor Perlaki ${ }^{1,2,3}$, Gergely Orsi ${ }^{1,2,3}$, Szilvia Anett Nagy ${ }^{1,2,3,4}$, Edit Bosnyák
} David Kamson Olayinka ${ }^{5}$, Flóra John ${ }^{5}$, Anita Trauninger ${ }^{5}$ and Zoltán Pfund ${ }^{5^{*}}$ (I)

\begin{abstract}
Background/Aim: Migraine-related intracerebral white matter lesions (WMLs) are likely to be microvascular in nature and can be found in all hemispheric lobes. The aim of this study was to investigate migraine patients with or without WMLs to see the effects of these tissue damages on cortical thickness and volume. The role of migraine characteristics (duration of headache, attack frequency, estimated lifetime attack number, aura) was also tested.

Methods: As study participants, 161 female migraine patients (63 with aura; 52 with WMLs) and 40 age-matched healthy female subjects were enrolled in the study. None of the included migraine patients' headache or aura (where present) was unilaterally side-locked. Patients and controls were all right handed. Except for migraine, patients were free of any medical comorbidity. Cortical reconstruction and segmentation were performed on the 3D T1-weighted images using Freesurfer 5.3 image analysis suite. The automatic cortical parcellation was based on Freesurfer's Desikan-KillianyTourville atlas, which has 31 cortical regions per hemisphere. The segmented regions were divided into five lobes (frontal, parietal, temporal, occipital, insula). Since the left and right differences in lobar and insular volumes/thicknesses were not different among our groups, volume and cortical thickness were calculated for corresponding bilateral lobes.

Results: There was no significant difference in age between the whole migraine and the control groups. Migraineurs with WMLs ( $L+$ patients) were significantly older than lesion-free $(L-)$ patients $(P=0.0003)$ and controls $(P=0.018)$. Disease duration ( $P=0.003)$, the total number of migraine attacks $(P=0.022)$ and the rate of aura $(P=0.0003)$ were significantly higher in $L+$ patients than in $L$ - patients. Cortical thickness and volume measurements of lobes were not statistically different between the three groups $(L+, L-$, control). Age showed a significant negative association with both thickness and volume in each examined lobe $(P<0.001)$. Intracranial volume (ICV) showed a significant positive association with all regional volumes $(P<0.001)$. There were no significant group*age, group ${ }^{*} \mid C V$, or age*ICV interactions. None of the migraine characteristics were selected by stepwise linear regression as significant predictors of cortical thickness or volume. Only age (for both thickness and volume) and ICV (for volume) were identified as significant predictors ( $P<0$. 001). When the $L+$ group was divided into two subgroups by median split of total and lobar lesion number and volume, the cortical measures did not show any significant difference between the groups with low vs. high lesion number/volume by stepwise linear regression.

\footnotetext{
* Correspondence: pfund.zoltan@pte.hu

${ }^{5}$ Department of Neurology, Medical School, University of Pécs, Pécs, Hungary

Full list of author information is available at the end of the article
} 
(Continued from previous page)

Conclusions: In a female migraine group, we found that the WMLs and clinical migraine characteristics have no effect on cortical thickness and volume of bilateral lobes. Lobar cortical thicknesses were equivalent within the range of $\pm 0.1 \mathrm{~mm}$. Only age and ICV proved to be significant predictors; the former for both cortical thickness and volume, while the latter for cortical volume.

Keywords: Migraine-related intracerebral white matter lesions, Migraine headache characteristics, Lobar cortical thickness and volume, Magnetic resonance imaging, Cortical reconstruction and segmentation, Age, Intracranial volume

\section{Introduction}

Cortical thickness is both a marker of neurological development and a reflection of cortical function [1,2]. The cerebral cortex contains high neuronal density, and its thickness varies from $1.5 \mathrm{~mm}$ to $5 \mathrm{~mm}$ [3]. Both the pyramidal neurons and the interneurons travel through the white matter within the hemisphere during prenatal brain development, and both types of cortical neuronal cells receive projection fibers from the thalamus, and association and commissural fibers from other cortical areas [3].

Migraine is a primary headache disorder [4] that may cause structural and functional alterations in the cerebral cortex [5-8]. Migraine-related intracerebral white matter lesions (WMLs) are likely to be microvascular in nature and can be found in all four lobes implicating the deep white matter, the subcortical, the periventricular and the callosal commissure locations [9-11]. Based on the above mentioned data, we hypothesized that the WMLs - areas of focal axonal and glial cell (astrocyte, oligodendrocyte, microglia) injuries in association with decreased intracellular energy metabolism due to impairment of mitochondria - may cause cortical changes in migraine. For that reason, we investigated migraine patients with or without WMLs to assess the effects of these tissue damages on cortical thickness and volume. In this respect, the potential role of migraine characteristics was tested, as well. Female patients were selected, because migraine is much more prevalent in adult women than men [12] and to avoid the gender-related differences (e.g., longer headache duration, higher intensity of attacks, more frequent nausea, phonophobia and photophobia in women) existing between women and men [13].

\section{Methods}

\section{Subjects}

Between 2010 and 2017, a total of 161 female patients fulfilling the International Headache Society (IHS) classification criteria for migraine with or without aura [4] were prospectively screened from the Outpatient Headache Clinic of the Department of Neurology, Medical School, University of Pécs, Hungary. At the time of the study period, all migraineurs had recurrent headaches, and none of them were on chronic prophylactic therapy. For acute migraine treatment, eletriptan, sumatriptan, ibuprofen, diclofenac, acetylsalicylic acid and/or acetaminophen were utilized. The demographic and clinical data of migraineurs were the following: mean age $39.3 \pm$ 12.5 , range $18-73$ years; disease duration $15.6 \pm 11.9$, range 1-57 years; attack frequency/month $5.6 \pm 4.5$, range $0.2-14.8$; total number of estimated lifetime migraine attacks (average monthly attack number $\times 12 \times$ number of migraine disease years to date) $966 \pm 1158$, range $12-6840 ; n=52$ with WMLs (L+ patients); $n=63$ with aura (Table 1). Migraineurs had no other types of headaches. None of the included migraine patients' headache or aura was unilaterally side-locked in nature. Magnetic resonance imaging (MRI) was performed in a headache-free period for each patient. Medical comorbidities that could influence migraine characteristics or lead to the formation of WMLs were excluded (hypertension, diabetes mellitus, kidney disease, hepatopathy, high LDL-cholesterol, hyperuricemia, elevated CRP level, thyroid gland disease, systemic autoimmune disease, smoking, cardiac source of embolism, obesity). Based on self-report, all migraineurs were right handed. As controls, 40 age-matched healthy female subjects were included (mean age 38.3 \pm 10.0 , range $19-66$ years, Table 1 ). Controls were recruited by family physicians in Baranya County, Hungary. Similar to migraine patients, all controls were right handed. All control subjects were free of headache, and their brain MRI studies did not show any structural abnormalities.

\section{MRI acquisition}

All subjects were scanned on the same $3 \mathrm{~T}$ MRI scanner (Magnetom TIM Trio, Siemens AG, Erlangen, Germany) using a 12-channel head coil. The MRI measurements of all patients were performed in a headache-free period. Whole-brain 3D magnetization-prepared rapid gradientecho (MPRAGE) was acquired using the following parameters: TR/TI $/ \mathrm{TE}=1900 / 900 / 3.4 \mathrm{~ms}$; bandwidth $=179 \mathrm{~Hz} /$ px; flip angle $=9^{\circ}$; $\mathrm{FOV}=210 \times 240 \mathrm{~mm}^{2}$, matrix size $=$ $224 \times 256$, slice thickness $=0.94 \mathrm{~mm}, 176$ axial slices. Beyond the routine T1- and T2-weighted measurements the scanning protocol also included 2D turbo spin-echo flui$d$-attenuated inversion recovery (FLAIR) imaging (TR/TI/ $\mathrm{TE}=13,200 / 2600 / 100 \mathrm{~ms} ; \quad$ bandwidth $=401 \mathrm{~Hz} / \mathrm{px} ;$ echo trains $=14 ; \mathrm{FOV}=186 \times 220 \mathrm{~mm}^{2}$, matrix size $=162 \times 192$, 
Table 1 Demographic and clinical data of migraine patients and healthy controls

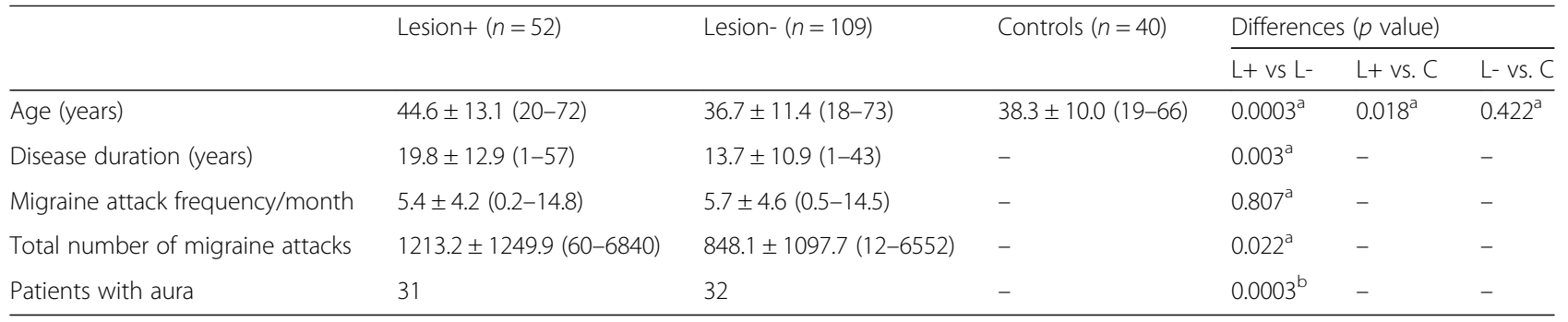

Lesion+/L+: Migraine patients with lesions; Lesion-/L-: Migraine patients without lesions; C: Control subjects; Values are given as mean \pm standard deviation (minimum-maximum); ${ }^{a}$ Mann-Whitney U-test; ${ }^{b}$ Fisher's exact test

slice thickness $=1.5 \mathrm{~mm}, 100$ axial slices). WML was considered if visible as hyperintensity on T2-weighted and FLAIR MRI but without hypointensity on T1-weighted MRI and larger than $3 \mathrm{~mm}$, appearing in at least two consecutive slices [14].

\section{MR image analysis}

Supratentorial WMLs were marked manually on the FLAIR images using 3D Slicer software (http://www.slicer.org, Version 4.6.2). An example of WML is displayed on Fig. 1. Total and lobar numbers/volumes of WMLs were calculated for each subject. The borders of lobes were defined as previously described [10].

Cortical reconstruction and segmentation were performed on the T1-weighted 3D MPRAGE images using Freesurfer 5.3 image analysis suite (https://surfer.nmr.mgh.harvard.edu/fswiki). The details of the image processing pipeline are described in prior publications $[15,16]$. Quality control was performed throughout the automatic processing stream. When the reconstruction was inaccurate, error correction was performed based on the recommended workflow (http://surfer.nmr.mgh.harvard.edu/fswiki/

RecommendedReconstruction).

The automatic cortical parcellation was based on Freesurfer's "Desikan-Killiany-Tourville" (DKT) atlas [17], which has 31 cortical regions per hemisphere. The segmented regions were divided into four lobes, and surface-area weighted average cortical thickness was calculated for each lobe using the following equation:

LobarThickness $=\frac{\text { Thickness }_{1} * \text { Area }_{1}+\ldots+\text { Thickness }_{n} * \text { Area }_{n}}{\text { Area }_{1}+\ldots+\text { Area }_{n}}$,

where "Area" means the surface area and 1..n is the index of left and right hemispheric cortical regions included in the given lobe. For the definition of frontal, parietal, temporal and occipital lobes please see Table 2. Lobar volumes were calculated as the sum of regional volumes from both hemispheres. Beyond the four lobes, insula was also investigated as the fifth hemispheric lobe of the brain [18]. Insular volume was defined as the sum of left and right insular volumes and mean insular thickness was calculated by averaging the thickness of the left and right insula. Cortical thicknesses and volumes were calculated from both hemispheres because of the following reasons: (i) we had no hypothesis on lateralized effects of migraine attacks (i.e., no migraine patient with unilateral side-locked headache or migraine aura), (ii) the left and right differences in lobar and insular volumes/thicknesses (i.e., lateralities) were not different among our three groups (see the Results section), (iii) we hypothesized a similar degree of white matter damage in the two hemispheres based on an earlier study reporting no differences between the left and right

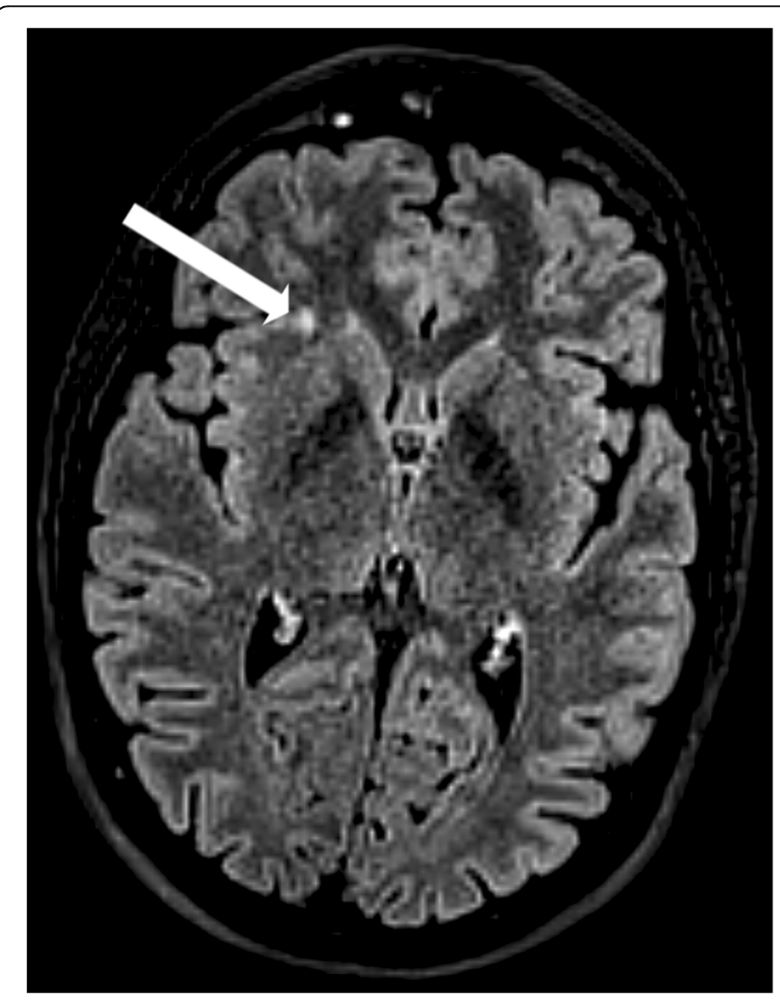

Fig. 1 White matter lesion of a migraine patients. The arrow shows a deep brain white matter lesion in the right frontal lobe seen on the axial fluid-attenuated inversion recovery image. The image is in radiological convention 
Table 2 Definition of lobes based on Freesurfer labels

\begin{tabular}{llll}
\hline Frontal & Parietal & Temporal & Occipital \\
\hline Superior Frontal & Superior Parietal & Superior, Middle, and Inferior Temporal & Lateral Occipital \\
Rostral and Caudal Middle Frontal & Inferior Parietal & Fusiform & Lingual \\
Pars Opercularis, Pars Triangularis, and Pars Orbitalis & Supramarginal & Transverse Temporal & Cuneus \\
Lateral and Medial Orbitofrontal & Postcentral & Entorhinal & Pericalcarine \\
Precentral & Precuneus & Parahippocampal & \\
Paracentral & Posterior Cingulate & \\
Rostral Anterior Cingulate & Isthmus Cingulate & \\
Caudal Anterior Cingulate & & \\
\hline
\end{tabular}

hemispheres in the number of WML, total WML volume, and average WML size in migraine [19].

\section{Statistical analysis}

Statistical analyses were performed using SPSS 20.0 software (IBM Corp., Armonk, NY). Differences in age between the whole migraine group and healthy controls were assessed by the Mann-Whitney U-test. Age differences among migraine subgroups ( $\mathrm{L}+$ and $\mathrm{L}-$ ) and the healthy control group were assessed by Kruskal-Wallis test followed by pair-wise comparison using the MannWhitney U-test. Continuous migraine-related variables (i.e., disease duration, migraine attack frequency, total number of migraine attacks) were compared between migraine subgroups ( $\mathrm{L}+$ and $\mathrm{L}-$ ) via the Mann-Whitney U-test, while differences in the rate of aura between the same subgroups were assessed using Fisher's exact test. The left and right differences in lobar and insular volumes/thicknesses (i.e., lateralities of volumes/thicknesses) were compared between our three groups $(\mathrm{L}+$, L-, control) using ANOVA. Cortical thickness and volume differences between the three groups ( $\mathrm{L}+, \mathrm{L}-$, control) were assessed using ANCOVA with age as covariate for the thickness, and age and total intracranial volume (ICV) as covariates for the volume. Concentrating on the whole migraine group, the possible effects of migraine characteristics (i.e., disease duration, migraine attack frequency, total number of migraine attacks, aura) on cortical thickness and volume were tested using stepwise multiple linear regression analyses. In these models age (for both thickness and volume) and ICV (for volume) were also included as possible predictors.

Concentrating on migraine subgroup with lesion $(L+)$, we assessed the potential effects of lesion number/volume on lobar and insular thicknesses/volumes. Unfortunately, all of our total and lobar lesion number/volume data were definitely not normally distributed (right-skewed with several extreme values). In order to perform powerful statistical analyses, L+ group was divided into patients with mild and patients with moderate-severe lesions. The division was performed based on the median split of whole brain as well as each lobar lesion number/volume, thereby creating binarized subgroup variables: e.g., subgroup with low (below or equal to the median) vs. subgroup with high number of frontal lobe lesions. Since lesions were rare in the occipital lobe with a median lesion number of 0 , the division based on lesion number/volume of this lobe was not performed. The effects of these subgroup variables on the insular and lobar thicknesses/volumes were tested using stepwise multiple linear regression analyses, including subgroup variables from the whole brain (for both insular and lobar thicknesses and volumes) and from the same lobe as that of dependent variable (for the lobar thicknesses volumes), age (for both thicknesses and volumes) and ICV (for volumes) as possible predictors. The level of statistical significance was set as $<0.05$.

\section{Results}

There was no significant difference in age between the whole migraine (including both $L+$ and $L$-patients) and the control groups $(P=0.738)$. The Kruskal-Wallis test revealed significant age differences among migraine subgroups (L+ and $\mathrm{L}-)$ and controls $(P=0.001)$. Post-hoc testing indicated that $\mathrm{L}+$ subgroup was significantly older than the L- $(P=0.0003)$ and the control $(P=0.018)$ groups (Table 1$)$. Disease duration $(P=0.003)$, the total number of migraine attacks $(P=0.022)$ and the rate of aura $(P=0.0003)$ were also significantly higher in $\mathrm{L}+$ patients than in L- patients (Table 1 ).

The left and right differences in lobar and insular volumes/thicknesses (i.e., lateralities) were not different among our groups (L+, L-, control); $P=0.626,0.965$, 0.425, 0.859 and 0.989 for the frontal, parietal, temporal, occipital and insular thicknesses and $P=0.598,0.252$, 0.855, 0.732 and 0.136 for the frontal, parietal, temporal, occipital and insular volumes, respectively. Cortical thickness and volume measurements of the five lobes were not statistically different among our three groups (L+, L-, control), (Table 3.). For both thickness and volume, the mean differences between our groups and the Bonferroni corrected 95\% confidence intervals of mean differences are presented in Table 4. 
Table 3 Group differences in cortical thickness and volume

\begin{tabular}{|c|c|c|c|c|c|c|c|c|c|}
\hline & \multicolumn{3}{|l|}{ Groups } & \multicolumn{6}{|c|}{ ANCOVA test } \\
\hline & \multirow[t]{2}{*}{ Lesion+ } & \multirow[t]{2}{*}{ Lesion- } & \multirow[t]{2}{*}{ Control } & \multicolumn{2}{|c|}{ Group effect } & \multicolumn{2}{|c|}{ Age effect } & \multicolumn{2}{|c|}{ ICV effect } \\
\hline & & & & $\mathrm{F}$ & $\mathrm{P}$ & $\mathrm{F}$ & $P$ & $\mathrm{~F}$ & $P$ \\
\hline Frontal thickness (mm) & $2.51(0.11)$ & $2.55(0.09)$ & $2.51(0.10)$ & 1.41 & 0.246 & 63.12 & $<0.001^{\mathrm{a}}$ & - & - \\
\hline Parietal thickness (mm) & $2.23(0.11)$ & $2.26(0.11)$ & $2.26(0.11)$ & 0.51 & 0.603 & 72.13 & $<0.001^{\mathrm{a}}$ & - & - \\
\hline Temporal thickness (mm) & $2.81(0.11)$ & $2.83(0.11)$ & $2.80(0.09)$ & 0.92 & 0.401 & 35.91 & $<0.001^{\mathrm{a}}$ & - & - \\
\hline Occipital thickness (mm) & $1.90(0.10)$ & $1.92(0.09)$ & $1.89(0.08)$ & 0.81 & 0.445 & 28.65 & $<0.001^{\mathrm{a}}$ & - & - \\
\hline Insula thickness (mm) & $3.05(0.14)$ & $3.09(0.14)$ & $3.06(0.12)$ & 0.23 & 0.796 & 29.35 & $<0.001^{\mathrm{a}}$ & - & - \\
\hline Frontal volume $\left(\mathrm{mm}^{3}\right)$ & $158,308(16892)$ & $163,774(15889)$ & 161,019 (15212) & 0.34 & 0.713 & 83.22 & $<0.001^{\mathrm{a}}$ & 92.04 & $<0.001^{b}$ \\
\hline Parietal volume $\left(\mathrm{mm}^{3}\right)$ & $103,341(11878)$ & $106,370(10566)$ & $106,016(8878)$ & 0.11 & 0.897 & 70.13 & $<0.001^{\mathrm{a}}$ & 69.83 & $<0.001^{\mathrm{b}}$ \\
\hline Temporal volume $\left(\mathrm{mm}^{3}\right)$ & $98,752(9514)$ & $102,308(8639)$ & $100,105(8294)$ & 1.71 & 0.184 & 36.60 & $<0.001^{\mathrm{a}}$ & 78.76 & $<0.001^{\mathrm{b}}$ \\
\hline Occipital volume $\left(\mathrm{mm}^{3}\right)$ & 40,789 (5254) & $42,196(4531)$ & $40,721(4414)$ & 1.23 & 0.294 & 33.85 & $<0.001^{\mathrm{a}}$ & 42.13 & $<0.001^{\mathrm{b}}$ \\
\hline Insula volume $\left(\mathrm{mm}^{3}\right)$ & $10,937(1066)$ & 11,039 (994) & 10,938 (995) & 0.28 & 0.758 & 24.25 & $<0.001^{\mathrm{a}}$ & 27.53 & $<0.001^{b}$ \\
\hline
\end{tabular}

Lesion+: Migraine patients with lesions; Lesion-: Migraine patients without lesions; ICV: total intracranial volume; All thicknesses/volumes were excluded from the analysis, where the corresponding standardized residuals from the ANCOVA model were below -3 or above 3 . Maximum three subjects had to be excluded from each group. Thicknesses and volumes are presented as uncorrected mean (standard deviation); ${ }^{a}$ negative (inverse) association with thickness/volume, ${ }^{b}$ positive association with volume

Age showed a significant negative association with both thickness and volume in each examined lobe $(P<$ 0.001). Intracranial volume showed a significant positive association with the volumes of all regions $(P<0.001)$. There were no significant group*age, group*ICV or age*ICV interactions in the performed analyses.

In the whole migraine group, none of the migraine characteristics were selected by stepwise linear regression as significant predictors of cortical thickness or volume. Only age (for both thickness and volume) and ICV (for volume) were identified as significant predictors $(P$ $<0.001)$.

Focusing on the L+ patients, none of the binarized total or lobar lesion number/volume variables were selected by stepwise linear regression as significant predictors of the insular or lobar thicknesses/volumes. The main features of WMLs in the L+ group are presented in Table 5.

\section{Discussion}

In this study, we investigated a homogeneous (female migraineurs without medical comorbidities) migraine group to explore the potential effects of WMLs and migraine characteristics on cortical lobar thickness and volume. The WMLs and clinical characteristics failed to show any effects on the lobar cortical measures. When the lesion + group was divided into two subgroups by median split of total and lobar lesion number and volume, the cortical measurements (thickness and volume) did not show any significant difference between the groups with low vs. high lesion number/volume by stepwise

Table 4 Differences in marginal means and 95\% confidence intervals

\begin{tabular}{|c|c|c|c|}
\hline & \multicolumn{3}{|c|}{ Mean Difference ${ }^{a}$ (95\% Confidence Interval for Difference ${ }^{b}$ ) } \\
\hline & $L+$ minus $L-$ & $L+$ minus $C$ & $L-$ minus $C$ \\
\hline Frontal thickness (mm) & $-0.0002(-0.037$ to 0.037$)$ & $0.026(-0.019$ to 0.072$)$ & $0.027(-0.013$ to 0.066$)$ \\
\hline Parietal thickness (mm) & $0.016(-0.024$ to 0.056$)$ & $0.006(-0.044$ to 0.055$)$ & $-0.010(-0.053$ to 0.032$)$ \\
\hline Temporal thickness (mm) & $0.010(-0.033$ to 0.052$)$ & $0.029(-0.023$ to 0.082$)$ & $0.019(-0.026$ to 0.065$)$ \\
\hline Occipital thickness (mm) & $0.002(-0.033$ to 0.038$)$ & $0.021(-0.023$ to 0.064$)$ & $0.018(-0.020$ to 0.056$)$ \\
\hline Insula thickness (mm) & $-0.004(-0.059$ to 0.051$)$ & $0.012(-0.055$ to 0.080$)$ & $0.016(-0.042$ to 0.075$)$ \\
\hline Frontal volume $\left(\mathrm{mm}^{3}\right)$ & $-1217(-5905$ to 3470$)$ & $200(-5539$ to 5938$)$ & $1417(-3527$ to 6361$)$ \\
\hline Parietal volume $\left(\mathrm{mm}^{3}\right)$ & $-270(-3560$ to 3020$)$ & $-767(-4795$ to 3260$)$ & -497 (- 3967 to 2972$)$ \\
\hline Temporal volume $\left(\mathrm{mm}^{3}\right)$ & $-1944(-4803$ to 915$)$ & $-336(-3836$ to 3164$)$ & $1608(-1407$ to 4623$)$ \\
\hline Occipital volume $\left(\mathrm{mm}^{3}\right)$ & $-422(-2073$ to 1229$)$ & 704 (- 1315 to 2724$)$ & 1126 (- 616 to 2868$)$ \\
\hline Insula volume $\left(\mathrm{mm}^{3}\right)$ & 84 ( -290 to 459$)$ & $139(-323$ to 600$)$ & $54(-346$ to 455$)$ \\
\hline
\end{tabular}

L+: Migraine patients with lesions; L-: Migraine patients without lesions; C: Control subjects

Differences in marginal means are presented as mean ( $95 \%$ confidence interval for the difference)

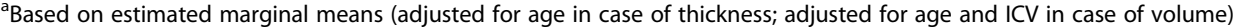

${ }^{\mathrm{b}}$ Adjustment for multiple comparisons: Bonferroni 
Table $\mathbf{5}$ Location, number and size of WMLs in the migraine group with lesions

\begin{tabular}{lll}
\hline Location & Number of WMLs & Total WML volume $\left(\mathrm{mm}^{3}\right)$ \\
\hline Frontal lobe & $6.5(2-11)$ & $168.4(66.0-447.1)$ \\
Parietal lobe & $2(0-7)$ & $54.2(0-318.5)$ \\
Temporal lobe & $0.5(0-2)$ & $8.9(0-42.3)$ \\
Occipital lobe & $0(0-1)$ & $0(0-22.2)$ \\
Whole brain & $10.5(3-19)$ & $338.0(119.1-963.2)$ \\
\hline
\end{tabular}

WML: white matter lesion; The number and volume of lesions failed the Shapiro-Wilk normality test, thus values are presented as median (25th -75th percentile); Total WML volume was calculated by the sum of individual WML volumes in the given lobe

linear regression. Only age and ICV proved to be significant predictors; the former for both cortical thickness and volume, while the latter for cortical volume.

The lack of the impact of WMLs on cortical thickness and volume may be the consequence of the not reaching the critical size of injured white matter territory, the intralobar separations of lesions with differences in distributions, or less severe intralesional tissue damage. Although the clinically silent brain WMLs are predominantly progressive in nature, smaller lesions may improve in size or even disappear [10]. In addition, the normal-appearing white matter (NAWM) did not show MRI signs of tissue injury in migraine patients with or without WML [9].

The negative association of age with cortical thickness and volume raises the possibility that WMLs are age-related. WMLs can develop at any age during the active migraine years, and their presence does not correlate with age [20-23]. Disease duration and attack frequency are the main indicators for brain damage in migraine [22, 24]. Usually, ageing associates with longer disease duration and higher lifetime attack number, and these factors may increase the risk of oxidative stress-related endothelial injury and atherosclerosis [11]. Furthermore, a wide range of vascular risk factors contribute to lesion formation [22, 25]. In the present study, both migraineurs and controls lacked any medical comorbidity, thus the role of ageing in lesion development is less likely.

Previous studies presented several different cortical regions with morphological abnormalities, sometimes with contradictory findings. Due to differences in study aims, morphometric MRI studies, analytical approaches and number of study participants, our results are not directly comparable to earlier studies $[6-8,26-36]$.

Some of these studies used a voxel-based morphometry (VBM) approach [7, 8, 26, 27, 29, 32, 36], which analyzes the brain on a voxel by voxel basis, while our approach examines brain changes at a larger territory level (i.e., lobar level). In addition, several other methodological differences exist between VBM and our surface-based analysis. VBM does not distinguish between different cortical morphological properties and various methodological factors, including cortical thickness, surface area, cortical volume, gyrification pattern, T1 signal alterations within a physiologic range, registration artefacts and smoothing [37-39]. Moreover, the significant group differences reported by a VBM analysis are not necessarily homogenous in terms of the underlying factors [37]. Based on these differences, VBM and our method should be considered as complementary techniques [38].

Others used surface-based analysis to examine local cortical thickness changes in migraine $(6,28,30,31,33-$ $35)$. Most of these studies were not interested in the direct thickness differences between migraineurs and controls, but rather examined between-hemisphere cortical differences related to the headache side [6], interregional cortical thickness correlations to differentiate groups of migraine patients from healthy controls [34], and differences in cortical thickness-to-pain threshold correlations between migraineurs and controls [33]. Another study reported significantly decreased left anterior midcingulate cortical thickness in the migraine group, but the number of subjects was low (17 migraine patients vs. 18 controls) [31]. From the largest studies, one reported no significant cortical thickness differences at all [28], while the other found significant differences between migraineurs and controls in several small brain regions [30]. The latter should be interpreted in the context of statistically controlling for the whole-hemisphere average cortical thickness during vertex-wise statistical analysis of thickness. Since the overall average cortical thickness was significantly increased in the migraineurs, such correction may add noise and provide inaccurate data. A vertex-based study conducted on females found higher cortical thickness in the superior frontal gyrus, paracentral gyrus, temporal pole, precuneus and lower cortical thickness in the anterior cingulate of migraineurs [25]. Interestingly, the authors were unable to detect the well-established cortical atrophy with advancing age [40] for the insula in the migraine group, while we could demonstrate it by measuring both cortical thickness and cortical volume without any group*age interaction. Two recent studies investigated only migraine patients with aura $[41,42]$. One of them found slightly thicker cortical visual areas in female migraineurs with aura [41], while the other found no solid evidence for cortical thickness differences between patients with aura and controls [42]. The second study found reduced volume of the left fusiform gyrus in migraineurs with aura compared to controls. We conducted a region-of-interest (ROI) surface-based analysis, which has unique strengths and limitations compared to the vertex-wise surface-based method (e.g., ROI analysis does not need smoothing or inter-subject registration, and the problem of multiple comparisons is substantially reduced compared to a vertex-wise comparison). The measurement at a single vertex is often quite noisy, which may reduce the statistical power [43]. However, if the structural differences 
cross the boundaries of the predefined ROIs, then only the vertex-wise approach may find them [44]. Moreover, the vertex-wise approach doesn't need an a priori hypothesis. From the above cited studies, only Datta et al. [28] and Gaist at al [41]. used a similar approach to our one, and although the results of Datta et al. [28] were also negative, the investigated ROIs were quite different in both of these studies compared to the present study.

In one study the cortical thickness measures by Freesurfer agreed to those obtained using traditional neuropathologic techniques within $0.2 \mathrm{~mm}$ (with a mean difference of $0.077 \mathrm{~mm}$ ) [45]; in another study the accuracy was better than $0.5 \mathrm{~mm}$ compared to manual measures on MRI data [46], while test-retest within-scanner error in local cortical thickness measurement was found to be about $0.12 \mathrm{~mm}$ in average [47]. Based on the order of these numbers, in the present study, only cortical thickness differences of at least $0.1 \mathrm{~mm}$ was considered scientifically relevant. Since our Bonferroni corrected 95\% confidence intervals of the cortical thickness differences were within the range of -0.059 to $+0.082 \mathrm{~mm}$ (Table 4) for each lobe and each subgroup comparison, equivalence testing suggests that mean lobar cortical thicknesses of all three groups are equivalent within the pre-defined practically relevant limits (i.e., $\pm 0.1 \mathrm{~mm}$ ).

\section{Strengths and limitations}

The main strengths of our study include the relatively large single center sample size, assessing both cortical thickness and volume, and performing equivalence testing. Our study was cross-sectional, where the detection of subtle cortical changes is hindered by inter-individual differences. Longitudinal studies eliminating much of these differences are also needed to further support that no cortical changes occur in migraine. Instead of searching for very subtle structural differences, our main goal was to examine whether migraine lesions cause thickness/volume changes at the level of larger cortical territories (i.e., lobar level). This approach is useful to detect more robust lobar changes even if there are individual variations of morphometric changes within the lobes. However, we acknowledge that very subtle structural changes with consistent anatomical locations can exist, which may be detected by vertex-wise analysis or more detailed ROI analysis, while such abnormalities may be averaged out and overlooked when examining at the lobar level.

\section{Conclusions}

In summary, we investigated a female migraine group, and found that neither the lesions nor other clinical characteristics have a detectable effect on cortical thickness and volume of bilateral intracerebral lobes. Cortical thicknesses were equivalent within the range of \pm 0.1 $\mathrm{mm}$. Only age and ICV proved to be significant predictors; the former for both cortical thickness and volume, while the latter for cortical volume.

\section{Abbreviations}

ANCOVA: Analysis of covariance; ANOVA: Analysis of variance; DKT: DesikanKilliany-Tourville; FLAIR: Fluid-attenuated inversion recovery; FOV: Field-ofview; ICV: Intracranial volume; IHS: International Headache Society; MPRAGE: Magnetization-prepared rapid gradient-echo; MRI: Magnetic resonance imaging; NAWM: Normal-appearing white matter; ROI: Region-ofinterest; SPSS: Statistical package for the social sciences; VBM: Voxel-based morphometry; WML: White matter lesions

\section{Acknowledgements}

Not applicable.

\section{Funding}

This work was supported by grants from EEA/Norwegian Financial Mechanism HU 0114 -"Save what can be saved" - applied neurological research using high-field magnetic resonance imaging, by TÁMOP-4.2.1/B, EFOP-3.6.2-16-2017-00008, "The role of neuroinflammation in neurodegeneration: From molecules to clinics", Hungarian Brain Research Program (KTIA_NAP_13-2-2014-0019 and 2017-1.2.1-NKP-2017-00002), Institutional Excellence Program (17886-4/2018/FEKUTSTRAT), ÚNKP-18-4-PTE-315 New National Excellence Program of the Ministry of Human Capacities, PTE ÁOKKA-2017-05, PTE ÁOK-KA-2017-06 and János Bolyai Research Scholarship of the Hungarian Academy of Sciences (G. P. and G. O.). Z. P. was funded by PTE ÁOK-KA-2017-23. EFOP-3.6.3-VEKOP_00009.

\section{Availability of data and materials}

All data and materials are available. All authors take full responsibility for the data and materials.

\section{Authors' contributions}

Category 1(a) Conception and design ZP, DOK, GP. (b) Acquisition of data ZP, GP, EB, AT. (c) Analysis and interpretation of data GP, GO, SAN, MH, HK. Category 2 (a) Drafting the manuscript ZP, DOK, GP. (b)Revising it for intellectual content (c) ZP, DOK, GP, AT, FJ. Category 3 (a) Final approval of the completed manuscript HK, MH, GP, GO, SAN, EB, DOK, FJ, AT, ZP.

Ethics approval and consent to participate

All subjects provided informed consent to study participation. Studies were performed in accordance with the approval (4022) of the Regional Research Ethics Committee of the Medical Center, Pécs.

Consent for publication

Publication is accepted by all authors.

Competing interests

The authors declare that they have no competing interests.

\section{Publisher's Note}

Springer Nature remains neutral with regard to jurisdictional claims in published maps and institutional affiliations.

\section{Author details}

${ }^{1}$ Pécs Diagnostic Center, Pécs, Hungary. ${ }^{2}$ Department of Neurosurgery, Medical School, University of Pécs, Pécs, Hungary. ${ }^{3}$ MTA-PTE Clinical Neuroscience MR Research Group, Pécs, Hungary. ${ }^{4}$ Neurobiology of Stress Research Group, Szentágothai Research Center, University of Pécs, Pécs, Hungary. ${ }^{5}$ Department of Neurology, Medical School, University of Pécs, Pécs, Hungary.

Received: 17 October 2018 Accepted: 2 January 2019

Published online: 10 January 2019

\section{References}

1. Jernigan TL, Trauner DA, Hesselink JR, Tallal PA (1991) Maturation of human cerebrum observed in vivo during adolescence. Brain 114:2037-2049 
2. Reiss AL, Abrams MT, Singer HS, Ross JL, Denckla MB (1996) Brain development, gender and IQ in children. A volumetric imaging study. Brain 119:1763-1774

3. Cerebrum, chapter 9, pages 350-369. In: Duus' Topical Diagnosis in Neurology: Anatomy, Physiology, Signs, Symptoms, 4th edition - By M. Baehr and M. Frotscher 2006. ISBN 3-13-612804-4 (GTV), ISBN 1-58890-215-3 (TNY)

4. Headache Classification Committee of the International Headache Society (IHS) (2013) The international classification of headache disorders, 3rd edition (beta version). Cephalalgia 33:629-808

5. Valfrè W, Rainero I, Bergui M, Pinessi L (2008) Voxel-based morphometry reveals gray matter abnormalities in migraine. Headache 48:109-117

6. Hougaard A, Amin FM, Hoffmann MB, Larsson HB, Magon S, Sprenger T, Ashina M (2015) Structural gray matter abnormalities in migraine relate to headache lateralization, but not aura. Cephalalgia 35:3-9

7. Jia Z, Yu S (2017) Grey matter alterations in migraine: a systematic review and meta-analysis. Neuroimage Clin 14:130-140

8. Neeb L, Bastian K, Villringer K, Israel H, Reuter U, Fiebach JB (2017) Structural gray matter alterations in chronic migraine: implications for a progressive disease? Headache 57:400-416

9. Aradi M, Schwarcz A, Perlaki G, Orsi G, Kovács N, Trauninger A, Kamson DO, Erdélyi-Bótor S, Nagy F, Nagy SA, Dóczi T, Komoly S, Pfund Z (2013) Quantitative MRI studies of chronic brain white matter hyperintensities in migraine patients. Headache 53:752-763

10. Erdélyi-Bótor S, Aradi M, Kamson DO, Kovács N, Perlaki G, Orsi G, Nagy SA, Schwarcz A, Dóczi T, Komoly S, Deli G, Trauninger A, Pfund Z (2015) Changes of migraine-related white matter hyperintensities after 3 years: a longitudinal MRI study. Headache 55:55-70

11. Erdélyi-Bótor S, Komáromy H, Kamson DO, Kovács N, Perlaki G, Orsi G, Molnár T, Illes Z, Nagy L, Kéki S, Deli G, Bosnyák E, Trauninger A, Pfund Z (2017) Serum L-arginine and dimethylarginine levels in migraine patients with brain white matter lesions. Cephalalgia 37:71-580

12. Vetvik KG, MacGregor EA (2017) Sex differences in the epidemiology, clinical features, and pathophysiology of migraine. Lancet Neurol 16:76-87

13. Bolay H, Ozge A, Saginc P, Orekici G, Uludüz D, Yalın O, Siva A, Bıçakçi Ş, Karakurum B, Öztürk M (2015) Gender influences headache characteristics with increasing age in migraine patients. Cephalalgia 3:792-800

14. Vermeer SE, Hollander M, van Dijk EJ, Hofman A, Koudstaal PJ, Breteler MMB (2003) Silent brain infarcts and white matter lesions increase stroke risk in general population. Stroke 34:1126-1129

15. Dale AM, Fischl B, Sereno MI (1999) Cortical surface-based analysis. I. Segmentation and surface reconstruction. Neuroimage 9:179-194

16. Fischl B, Dale AM (2000) Measuring the thickness of the human cerebral cortex from magnetic resonance images. Proc Natl Acad Sci U S A 97: 11050-11055

17. Klein A, Tourville J (2012) 101 labeled brain images and a consistent human cortical labeling protocol. Front Neurosci 6:171

18. Stephani C, Fernandez-Baca Vaca G, Maciunas R, Koubeissi M, Lüders HO (2011) Functional neuroanatomy of the insular lobe. Brain Struct Funct 216:137-149

19. Kamson DO, Illés Z, Aradi M, Orsi G, Perlaki G, Leél-Őssy E, Erdélyi-Botor S, Pótó L, Trauninger A, Pfund Z (2012) Volumetric comparisons of supratentorial white matter hyperintensities on FLAIR MRI in patients with migraine and multiple sclerosis. J Clin Neurosci 19:696-701

20. Fazekas F, Koch M, Schmidt R, Offenbacher $H$, Payer F, Freidl W, Lechner $H$ (1992) The prevalence of cerebral damage varies with migraine type: a MRI study. Headache 32:287-291

21. De Benedittis G, Lorenzetti A, Sina C, Bernasconi V (1995) Magnetic resonance imaging in migraine and tension-type headache. Headache 35:264-268

22. Trauninger A, Leél-Ossy E, Kamson DO, Pótó L, Aradi M, Kövér F, Imre M, Komáromy H, Erdélyi-Botor S, Patzkó A, Pfund Z (2011) Risk factors of migraine-related brain white matter hyperintensities: an investigation of 186 patients. J Headache Pain. 12:97-103

23. Eidlitz-Markus T, Zeharia A, Haimi-Cohen Y, Konen O (2013) MRI white matter lesions in pediatric migraine. Cephalalgia 33:906-913

24. Schmitz N, Admiraal-Behloul F, Arkink EB, Kruit MC, Schoonman GG, Ferrari MD, van Buchem MA (2008) Attack frequency and disease duration as indicators for brain damage in migraine. Headache 48:1044-1055

25. Sacco S, Cerone D, Carolei A (2008) Comorbid neuropathologies in migraine: an update on cerebrovascular and cardiovascular aspects. J Headache Pain 9:237-248

26. Matharu MS, Good CD, May A, Bahra A, Goadsby PJ (2003) No change in the structure of the brain in migraine: a voxel-based morphometric study. Eur J Neurol 10:53-57
27. Rocca MA, Ceccarelli A, Falini A, Colombo B, Tortorella P, Bernasconi L, Comi G, Scotti G, Filippi M (2006) Brain gray matter changes in migraine patients with T2-visible lesions: a 3-T MRI study. Stroke 37:1765-1770

28. Datta R, Detre JA, Aguirre GK, Cucchiara B (2011) Absence of changes in cortical thickness in patients with migraine. Cephalalgia 31:1452-1458

29. Liu J, Zhao L, Li G, Xiong S, Nan J, Li J, Yuan K, von Deneen KM, Liang F, Qin W, Tian J (2012) Hierarchical alteration of brain structural and functional networks in female migraine sufferers. PLoS One 7:e51250

30. Messina R, Rocca MA, Colombo B, Valsasina P, Horsfield MA, Copetti M, Falini A, Comi G, Filippi M (2013) Cortical abnormalities in patients with migraine: a surface-based analysis. Radiology 268:170-180

31. Hubbard CS, Khan SA, Keaser ML, Mathur VA, Goyal M, Seminowicz DA (2014) Altered Brain Structure and Function Correlate with Disease Severity and Pain Catastrophizing in Migraine Patients. eNeuro 1:e20 14

32. Rocca MA, Messina R, Colombo B, Falini A, Comi G, Filippi M (2014) Structural brain MRI abnormalities in pediatric patients with migraine. J Neurol 261:350-357

33. Schwedt TJ, Chong CD (2014) Correlations between brain cortical thickness and cutaneous pain thresholds are atypical in adults with migraine. PLoS One 9:e99791

34. Schwedt TJ, Berisha V, Chong CD (2015) Temporal lobe cortical thickness correlations differentiate the migraine brain from the healthy brain. PLOS One 10:e0116687

35. Maleki N, Barmettler G, Moulton EA, Scrivani S, Veggeberg R, Spierings EL, Burstein R, Becerra L, Borsook D (2015) Female migraineurs show lack of insular thinning with age. Pain 156:1232-1239

36. Messina R, Rocca MA, Colombo B, Teggi R, Falini A, Comi G, Filippi M (2017) Structural brain abnormalities in patients with vestibular migraine. J Neurol 264:295-303

37. Palaniyappan L, Liddle PF (2012) Differential effects of surface area, gyrification and cortical thickness on voxel based morphometric deficits in schizophrenia. Neurolmage 60:693-699

38. Gerrits NJ, van Loenhoud AC, van den Berg SF, Berendse HW, Foncke EM, Klein M, Stoffers D, van der Werf YD, van den Heuvel OA (2016) Cortical thickness, surface area and subcortical volume differentially contribute to cognitive heterogeneity in Parkinson's disease. PLoS One 11:e0148852

39. Chung S, Wang X, Lui YW (2017) Influence of T1-weighted signal intensity on FSL voxel based Morphometry and FreeSurfer cortical thickness. AJNR Am J Neuroradiol 38:726-728

40. Salat DH, Buckner RL, Snyder AZ, Greve DN, Desikan RS, Busa E, Morris JC, Dale AM, Fischl B (2004) Thinning of the cerebral cortex in aging. Cereb Cortex 14:721-730

41. Gaist D, Hougaard A, Garde E, Reislev NL, Wiwie R, Iversen P, Madsen CG, Blaabjerg M, Nielsen HH, Krøigård T, Østergaard K, Kyvik KO, Hjelmborg J, Madsen K, Siebner HR, Ashina M (2018) Migraine with visual aura associated with thicker visual cortex. Brain. https://doi.org/10.1093/brain/awx382 [Epub ahead of print]

42. Petrusic I, Dakovic M, Kacar K, Zidverc-Trajkovic J (2018) Migraine with Aura: surface-based analysis of the cerebral cortex with magnetic resonance imaging. Korean J Radiol 19:767-776

43. Greve DN, Svarer C, Fisher PM, Feng L, Hansen AE, Baare W, Rosen B, Fischl B, Knudsen GM (2014) Cortical surface-based analysis reduces bias and variance in kinetic modeling of brain PET data. Neurolmage 92:225-236

44. Greve DN, Van der Haegen L, Cai Q, Stufflebeam S, Sabuncu MR, Fischl B, Brysbaert M (2013) A surface-based analysis of language lateralization and cortical asymmetry. J Cogn Neurosci 25:1477-1492

45. Rosas HD, Liu AK, Hersch S, Glessner M, Ferrante RJ, Salat DH, van der Kouwe A, Jenkins BG, Dale AM, Fischl B (2002) Regional and progressive thinning of the cortical ribbon in Huntington's disease. Neurology 58:695-701

46. Kuperberg GR, Broome MR, McGuire PK, David AS, Eddy M, Ozawa F, Goff D, West WC, Williams SC, van der Kouwe AJ, Salat DH, Dale AM, Fischl B (2003) Regionally localized thinning of the cerebral cortex in schizophrenia. Arch Gen Psychiatry 60:878-888

47. Han X, Jovicich J, Salat D, van der Kouwe A, Quinn B, Czanner S, Busa E, Pacheco J, Albert M, Killiany R, Maguire P, Rosas D, Makris N, Dale A, Dickerson B, Fischl B (2006) Reliability of MRI-derived measurements of human cerebral cortical thickness: the effects of field strength, scanner upgrade and manufacturer. Neurolmage 32:180-194 